\title{
Collagen changes in rheumatoid synovium
}

\author{
C. R. LOVELL \\ From the Department of Medicine, University of Bristol
}

The synovium is normally a single or double layer of cells with a thin weave of underlying connective tissue. In rheumatoid disease the synovium becomes swollen and congested, forming villous processes. Uitto et al. have demonstrated increased protocollagen proline hydroxylase activity (1970b) and a concomitant increase in collagen synthesis (1972) in rheumatoid as compared with normal synovium. Probably this inflammatory proliferation of synovial collagen forms an integral part of the disease process. We thought it important, therefore, to investigate the structure of collagen in normal synovium and the changes which occur in rheumatoid disease. This paper describes our findings concerning the proportions of type I and type III collagen in normal and rheumatoid synovium and the nature of the intermolecular cross-links. Finally, the effect of D-penicillamine on rheumatoid synovial collagen will be discussed.

\section{Investigations and results}

We collected rheumatoid material from 10 patients at synovectomy and dissected normal synovium from the knees of nine cadavers of ages ranging between three days and 96 years. Normal synovium was dissected mostly from the infrapatellar fat pad. We also took samples from the suprapatellar bursa and other areas of the knee joint, taking care to avoid the inclusion of other connective tissue structures such as joint capsule. The synovium was dissected free of adhering fat and capsule.

In order to elucidate the different molecular types of collagen we digested samples of synovium with pepsin at $4^{\circ} \mathrm{C}$ for 18 hours according to the method of Chung and Miller (1974). We confirmed the findings of Weiss et al. (1974) that rheumatoid synovium is more susceptible than normal to pepsin digestion under these conditions (about $60 \%$ compared with $5 \%$ solubilization). We separated the molecular types of collagen on the basis of molecular weight on SDS acrylamide gel electrophoresis. The gels were stained with Coomassie blue, destained, and scanned with a Joyce-Loëbl Chromoscan. The high proportion of $\gamma \mathrm{I}$ (III) component demonstrates the presence of type III collagen which is converted to $\alpha$ I (III) after incubation with $2 \%$ mercaptoethanol. An identical pattern was obtained with both normal and rheumatoid synovium.

However, pepsin solubilizes only a portion of the synovium, which might be atypical of the whole. Cyanogen bromide $(\mathrm{CNBr})$ cleaves collagen $\alpha$ chains at each methionine residue and different $\alpha$ chains give rise to characteristic peptides which can be separated by SDS acrylamide gel electrophoresis. This technique has the advantage that almost all the collagen is digested by cyanogen bromide. Examination of the $\mathrm{CNBr}$ peptides of both normal and rheumatoid synovium showed similar patterns, indicating the presence of both type I and type III collagens. Finally, rheumatoid synovium incubated in the presence of radioactive proline in Dulbecco's medium synthesized both types of collagen.

Thus normal synovium contains type I and type III collagens in about equal amounts and the proportion is unaltered in rheumatoid disease. It is unknown whether the two types of collagen are synthesized by two distinct clones of fibroblast.

\section{Effect of D-penicillamine}

The tensile strength of collagen in vivo is dependent on intermolecular cross-links. As previously described, the cross-links in young tissues are reducible and can be labelled with tritium by reduction with sodium ${ }^{3} \mathrm{H}$-borohydride (Bailey, 1970). Young normal synovium yields a high proportion of dihydroxylysinonorleucine (diOH-LNL) derived from the keto cross-link and a lesser amount of hydroxylysinonorleucine (OH-LNL) derived from the aldimine cross-link. In contrast with other connective tissues, such as skin and tendon, these reducible cross-links are present in lower but still appreciable amounts in elderly subjects. This suggests the presence of newly formed collagenthat is, a higher turnover rate than skin, etc. In rheumatoid synovium, however, hydroxylysino-5keto-norleucine (OHL-5-keto-NL) is again the dominant component.

D-penicillamine has been shown to suppress the rapid proliferation of the dermal plaque in scleroderma by inhibition of collagen synthesis. It also softens the skin by cleavage of the intermolecular 
cross-link (dehydro-OH-LNL) (Herbert et al., 1974). In rheumatoid synovium, however, the stabilizing cross-link is OHL-5-keto-NL, which is resistant to cleavage by $\mathrm{D}$-penicillamine. Similarly, incubation of shredded rheumatoid synovium for 24-48 hours with ${ }^{3} \mathrm{H}$-proline failed to show any alteration of collagen synthesis by the addition of D-penicillamine. Thus, despite its clinical efficacy in rheumatoid disease, we could find no evidence that $\mathrm{D}$-penicillamine has a direct effect on rheumatoid synovial collagen.

I thank Dr. Allen Bailey for his continued advice and encouragement and Angela Swan and Marie Gibson for expert technical assistance. This work was performed with the aid of a grant from the Dr. Hadwen Trust, for which I am most grateful. 